\title{
Substation Remote Viewing Image Mosaic based on Canny Operator and Harris Corner Detection
}

\author{
Yufan Wang \\ Hebei Software Institute \\ Hebei Baoding, 071003, China \\ E-mail: wangyufantx2004@126.com
}

\begin{abstract}
In order to improve the effect of substation operation remote video monitoring system, a new image mosaic method based on Canny operator and Harris corner detection is presented. Firstly, the edge feature points from two images are extracted by Canny edge detection; secondly, the corner feature points are extracted by Harris corner detection based on edge images; thirdly, the corresponding feature point pairs are got by the normalized cross correlation method around the corner feature points; finally, the images can be stitched by matched corner feature point pairs. The experiment of remote viewing image mosaic in substation shows that this method can achieve image mosaic effectively and it will be benefited to improve the safety and reliability of substation operation.
\end{abstract}

Keywords-Substation Remote Video Monitoring System; Image Mosaic; Canny Operator; Harris Corner Detection

\section{INTRODUCTION}

Current remote video monitoring system in substation cannot fully play its due role in real-time active supervision $[1,2,3,4]$. In order to improve the effect of remote video monitoring system, good mosaic technology must be presented. For a complete surveillance image, researchers must use image mosaic technology to piece the surveillance images together. Currently image mosaic field of image processing has become an important research aspect.

Image mosaic method involves two main steps: image registration and image fusion. The core technology of image mosaic is image registration technique. Current mainstream image mosaic method is feature-based image registration [5]. The basic image features includes region, texture, contour, edge and corner, etc. Edge and corner are the most basic features of all. But the existing methods are usually not ideal, they use edge features or corner features separately. Although the edges and corners have different features, there are also some relations. The corner of the image is usually the edge of the image, and is the intersection of two or more edges. If researchers can use the relation between the edge and corner points, researchers will obtain the feature points which are edge features and corner features meanwhile. Therefore, in this paper researchers present a new image mosaic method based on canny operator and Harris corner detection, which can improve the image registration accuracy and reduce the computation amount of image registration.

\section{EDGE DETECTION BASED ON CANNY OPERATOR}

Edge detection, especially step edge detection has been widely applied in various different computer vision systems, which is an important technique to extract useful structural information from different vision objects and dramatically reduce the amount of data to be processed. The Process of Canny edge detection algorithm can be broken down to 5 different steps:

1. Apply Gaussian filter to smooth the image in order to remove the noise.

2. Find the intensity gradients of the image.

3. Apply non-maximum suppression to get rid of spurious response to edge detection.

4. Apply double threshold to determine potential edges.

5. Track edge by hysteresis: Finalize the detection of edges by suppressing all the other edges that are weak and not connected to strong edges.

Every step has be described in details in article [6,7]. Figure 1-b is the edge image of Figure 1-a by Canny operator. Researchers can see that it has a good effect. In this paper, firstly researchers get the edge points of two images by Canny detection.

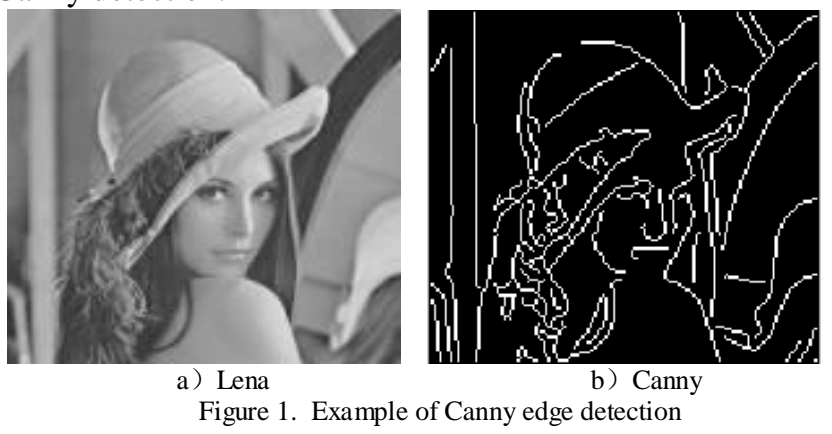

\section{CANNY HARRIS CORNER DETECTION UESING EDGE FEATURE POINTS}

Corner detection is an approach used within computer vision systems to extract certain kinds of features and infer the contents of an image. Harris and Stephens[8] presented the Harris corner detector, which is based on an underlying assumption that corners are associated with maxima of the local autocorrelation function. The Harris corner is computed as follows. 
1. Calculate the intensity $\mathrm{x}$-gradient $I_{\mathrm{x}}$ and the intensity y-gradient $I_{\mathrm{y}}$ bye using $3 \times 3$ convolution masks.

2. Eigenvalues or the determinant and trace of the gradient information matrix $H$ are computed to obtain the Harris response $R$. The matrix $H$ is defined as shown in article [9]. The Harris response $R$ can be calculated by formula (1).

$$
R=\operatorname{det} H-\alpha(\text { trace } H)^{2}
$$

3. A pixel $(x, y)$ is declared a Harris corner if the value $\mathrm{R}(x, y)$ is below a certain threshold.

In this paper, researchers get the corner points of two images by Harris corner detection from the Canny edge images, and researchers call it Canny Harris corner detection $(\mathrm{CH})$. Researchers chose two surveillance images in one current remote video monitoring system of substation. The
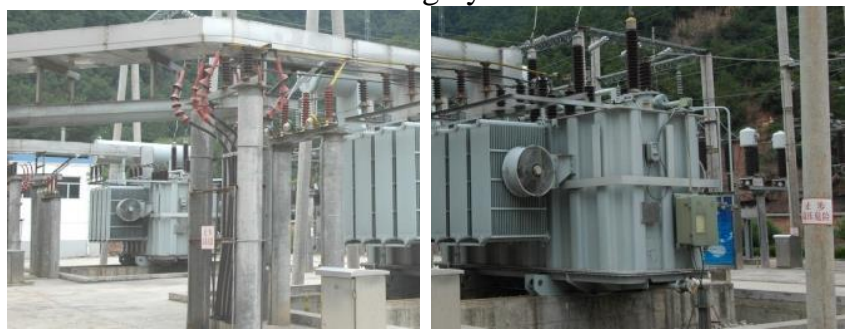

a) Surveillance images
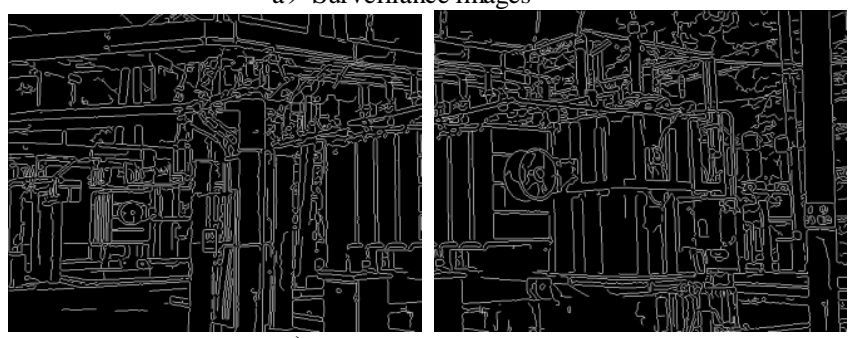

c) Canny edge detection

Figure

IV. IMAGE REGISTRATION AND IMAGE FUSION

After researchers get the corner feature points, researchers will use the normalized cross correlation method (NCC) [10] to get the corresponding feature point pairs. Firstly, calculate the correlation coefficients for all feature points between the two images, and set a threshold correlation coefficient $t$. If the correlation coefficient $K$ between feature points $p_{i}$ and $p_{i}^{\prime}$ of the two images is greater than the correlation coefficients between feature points $p_{i}$ and all points of one image, and meanwhile $K$ must be greater than threshold correlation coefficient $t$. Meet the above twoway matching consistency constraints, feature points $p_{i}$ and $p_{i}^{\prime}$ will be identified as the matching points of Harris corner in preparation for the next image registration work.

After matching point pairs, the two images are determined, let an image as a reference image. Image transformation matrix is calculated by matching point pairs, and two images are mapped to the same coordinate system by the transformation matrix. This image conversion model can be expressed in the form of a matrix, such as the formula (2). In formula (2), $H$ is the transformation matrix, $(x, y)$ two images are related to the same transformer, but the two images can not show the whole picture of the transformer. Therefore, if researchers want to get the whole picture of the transformer, researchers must stitch the two images. The size of image 1 is $551 \times 428$, and the size of image 2 is $558 \times 428$, as shown in Figure $2-a$. Firstly, researchers get the corner feature points based on general Harris corner detection method, and the results are in Figure 2-b. Secondly, researchers get the edge feature points by canny detection, as shown in Figure 2-c. Finally, researchers get the corner feature points by Canny Harris corner detection, as shown in Figure 2-d. Researchers can find Canny Harris corner detection get more corner points than General Harris corner detection, and these corner points are edge features and corner features meanwhile.
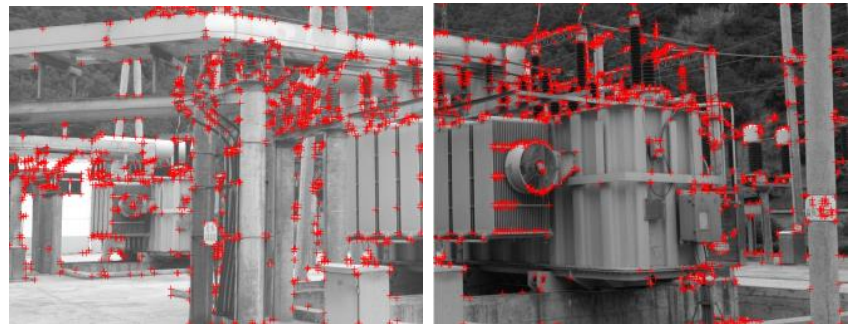

b) General Harris corner detection

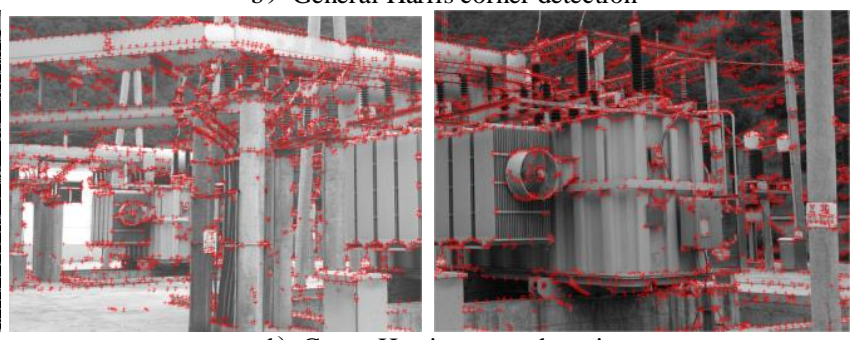

d) Canny Harris corner detection

and $\left(x^{\prime}, y^{\prime}\right)$ are separately the pixel coordinates of matching points in the reference image and the corresponding image. $k$ is the scale factor which reflect the relationship between images.

$$
k\left[\begin{array}{l}
x \\
y \\
z
\end{array}\right]=\left[\begin{array}{ccc}
m_{0} & m_{1} & m_{2} \\
m_{3} & m_{4} & m_{5} \\
m_{6} & m_{7} & 1
\end{array}\right]\left[\begin{array}{l}
x^{\prime} \\
y^{\prime} \\
1
\end{array}\right]=H\left[\begin{array}{l}
x^{\prime} \\
y^{\prime} \\
1
\end{array}\right]
$$

But in the real image registration process, there are some uncertainties, and the situations of mismatch may exist. Therefore, in order to improve the accuracy of image registration, researchers should be possible to eliminate the error matching points. In this paper, random sample consensus calculation (RANSAC) [11] is used to eliminate false matching pairs of points. RANSAC is computed as follows.

1. Four matching point pairs are randomly selected to form a random sample, and using LSM method to calculate transformation matrix $H$.

2. Based on transformation matrix $H$ and formula (3), the error geometric distance of each matching point 
pair is calculated. Where $\|$.$\| represents the Euclidean$ distance, $X_{1 i}$ and $X_{2 i}$ constitute a set of matching point pair. According to the transformation matrix $H$ researchers can calculate the projected points $X_{1 i}^{\prime}$ and $X_{2 i}^{\prime}$. When the distance dis by formula (3) is less than the threshold $t$, it is determined as the interior point, while the number of the interior points $\mathrm{cou}$ are calculated.

dis $=d\left(X_{1 i}, X_{2 i}^{\prime}\right)+d^{\prime}\left(X_{2 i}, X_{1 i}^{\prime}\right)=\|\| X_{1 i}-H X_{2 i}\|+\| x_{2 i}-H^{-1} X_{1 i} \|$ (3)

3. Repeat the above two steps, and choose the matrix $H$ with a maximum number of points as the transformation matrix. When there are an equal number of points in the case, select the matrix $H$ with the smallest standard deviation.

4. Iterative transformation matrix $H$, and re-estimate $H$.

5. Define the search area near the line of poles by the re-estimated $H$.

Firstly researchers get matching point pairs of two image in Figure.2-a by NCC based on general Harris corner detection, as shown in Figure.3-a. There are many false matching point pairs, so researchers use RANSAC to eliminate false matching point pairs, and the result is in Figure 3-b. Secondly, researchers get matching point pairs of two image by NCC based on Canny Harris $(\mathrm{CH})$ corner detection, as shown in Figure.3-c. After that, researchers use RANSAC to eliminate false matching point pairs, and the result is in Figure.3-d. Researchers can find the number of pairs by $\mathrm{CH}$ is less than by general Harris corner detection, so the $H$ by $\mathrm{CH}$ is more accurate.

After correct matching point pairs are gotten, researchers can use the transformation matrix $H$ to map the two images into a new image.

\section{EXPERIMENT OF IMAGE MOSAIC}

In this paper, researchers take two experiment of image mosaic. Firstly, researchers splice the images in Figure 2-a by above $\mathrm{CH}$ method. Researchers get the result of image mosaic, as shown in Figure.3-e.

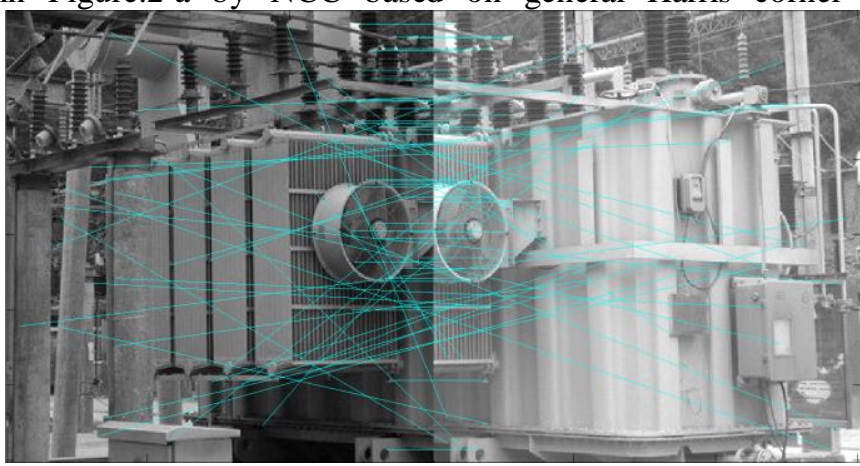

a) Result after NCC by general Harris
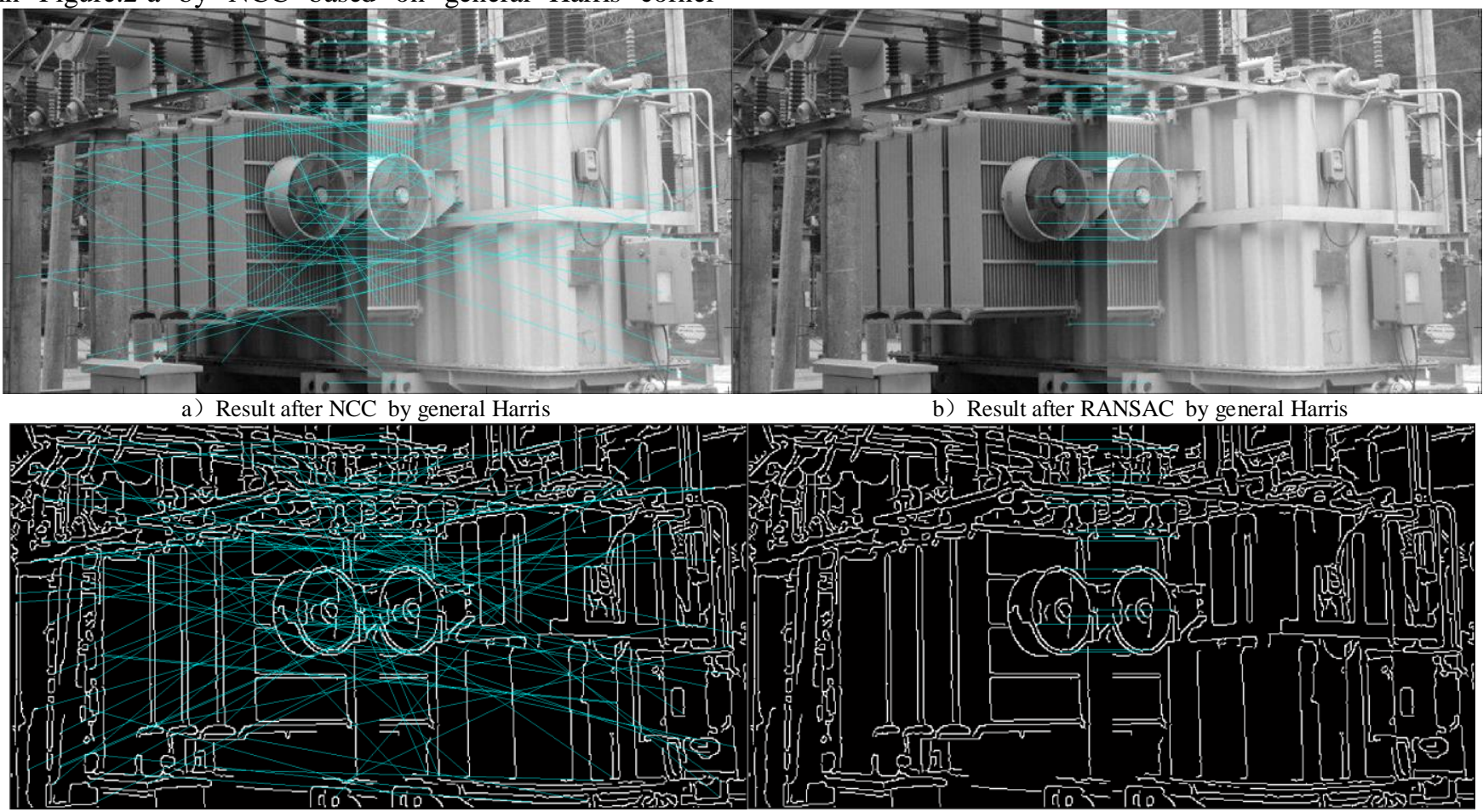

c) Result after NCC by Canny Harris corner detection b) Result after RANSAC by general Harris

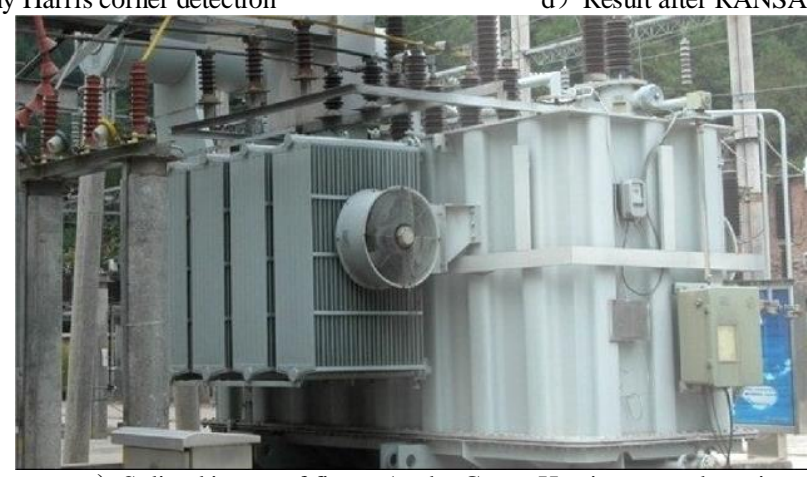

e) Spliced image of figure 1-a by Canny Harris corner detection

Figure 3. Example of Canny Harris corner detection 1 
Secondly, researchers splice the images in Figure 4-a by $\mathrm{CH}$ too. researchers get matching point pairs of two image by NCC based on Canny Harris $(\mathrm{CH})$ corner detection, as shown in Figure 4-b. After that, researchers use RANSAC to eliminate false matching point pairs, and the result is in Figure 4-c. The final result of image mosaic is in Figure 4-d.

The results of image mosaic in these experiments show that $\mathrm{CH}$ method can achieve image mosaic effectively and it is suitable for image mosaic needs in substation remote video
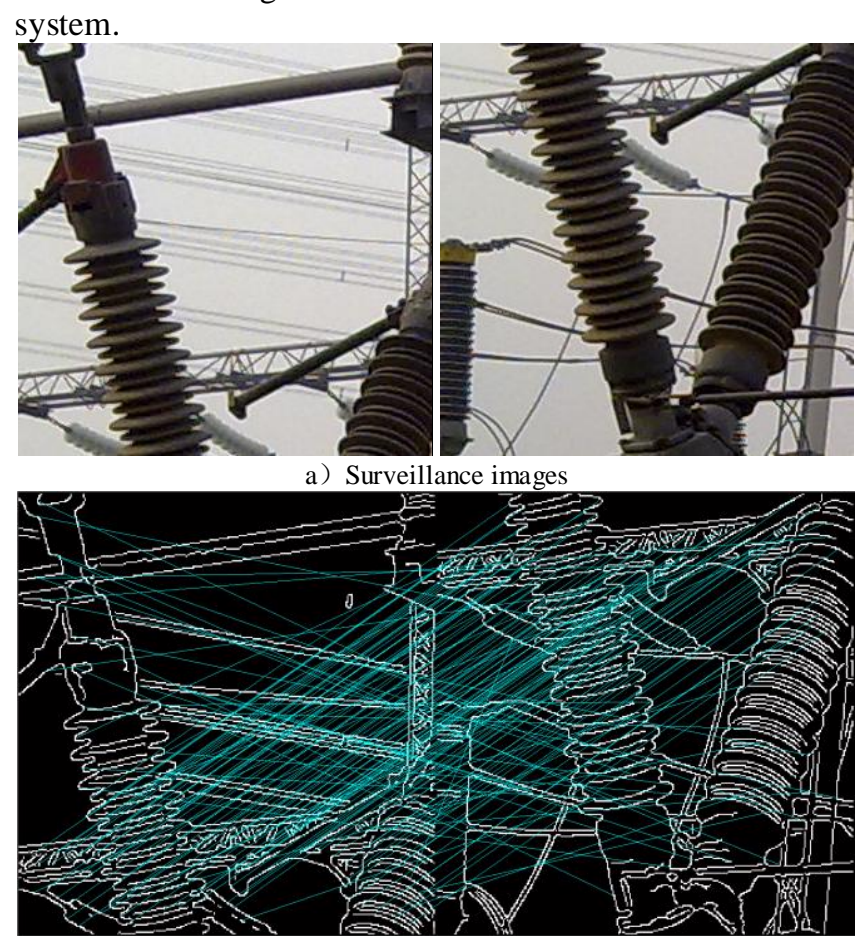

b) Result after NCC by Canny Harris corner detection

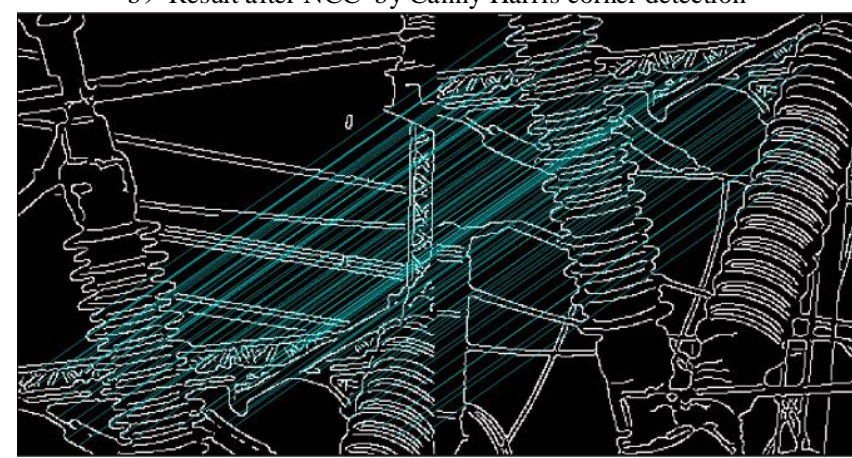

c) Result after RANSAC by Canny Harris corner detection

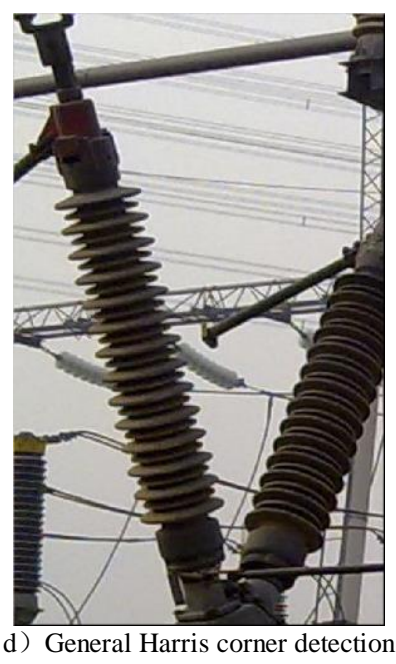

Figure 4. Example of Canny Harris corner detection 2

\section{CONCLUSIONS}

In this paper, researchers present a new image mosaic method based on Canny operator and Harris corner detection. Firstly, researchers use Canny edge detection to extract the edge points; secondly, the corner are extracted by Harris corner detection base on edge images; thirdly, the transformation matrix $H$ is calculated by NCC and RANSAC; finally, the new spliced image is gotten by the transformation matrix $H$. The experiments of remote viewing image mosaic in substation show that this method can achieve image mosaic effectively and improve the safety and reliability of substation operation.

\section{REFERENCES}

[1] G. Q. Xu, Y. X. Shen, Y. F. Zhang, "Study of digital video remote monitor system for unattended substation," Proceedings of the CSUEPSA, vol. 17, pp. 49-52, 2005.

[2] H. T. Zai, "Substation remote viewing image mosaic based on fuzzy cellular automata detection," Proceeding of the ICMSCM, pp. 12131216, 2013.

[3] S. Z. Hou, X. C. Tian, X. Lu, "Design and research of video supervisory control system of the substation," Relay, vol. 35, pp. 6064, 2007.

[4] Y. Y. Sang, S. L. Zheng, "Investigation and realization of Tele-Video system for provincial substation," Proceedings of the CSU-EPSA, vol. 21, pp. 73-77, 2009.

[5] X. Che, "Image mosaic algorithm based on feature points automatching," Optics \& Optoelectronic Technology, vol. 17, pp. 41-44, 2009.

[6] J. Canny, "A computational approach to edge detection," IEEE Trans. Pattern Analysis and Machine Intelligence, vol. 8, pp. 679-698, 1986.

[7] R. Ramya, P. S. Babu, "Automatic tuberculosis screening using canny Edge detection method," Proceeding of theICECS, pp. 282-285, 2015.

[8] C. Harris, M. J. Stephens, "Acombined corner and edge detector," Proceeding of the Alvey Vision Conf., pp. 869-872, 1988.

[9] J. Paul, W. Stechele, "Self-adaptive harris corner detector on heterogeneous many-core processor," Proceeding of the DASIP, pp. 8-13, 2014.

[10] M. T. Gao, S. R. Qu, X. Q. Li, "The theory and method of projection in computer vision research," Northwestern polytechnical University Press, Xi'an, 1998. 
[11] M. A. Fischler,R. C. Bolles, "Random Sample Consensus: A Paradigm for Model Fitting with Applications to Image Analysis and
Automated Cartography," Comm. of the ACM, vol. 24, pp. 381-395,

1981. 